\title{
Desafios da Engenharia de Requisitos Ágeis Centrada no Usuário
}

\author{
Nathália M. do Nascimento \\ Programa de Pós-graduação em \\ Informática (PPGI) / Universidade \\ Federal do Rio de Janeiro (UFRJ) \\ Rio de Janeiro, RJ - Brasil \\ nathalia_miranda@ufrj.br
}

\author{
Adriana S. Vivacqua \\ Programa de Pós-graduação em \\ Informática (PPGI) / Universidade \\ Federal do Rio de Janeiro (UFRJ) \\ Rio de Janeiro, RJ - Brasil \\ avivacqua@ppgi.ufrj.br
}

\author{
Mônica F. da Silva \\ Programa de Pós-graduação em \\ Informática (PPGI) / Universidade \\ Federal do Rio de Janeiro (UFRJ) \\ Rio de Janeiro, RJ - Brasil \\ monica.silva@ppgi.ufrj.br
}

\section{RESUMO}

Nos últimos anos, o interesse em desenvolver pesquisas sobre a integração de User centered design (UCD) e desenvolvimento de software ágil vem aumentando, mas por mais que os métodos ágeis tenham pontos de concordância com o UCD, também existem diferenças e essas diferenças ainda são um desafio em projetos de desenvolvimento. Ademais obter um entendimento completo dos requisitos do usuário é geralmente visto como incompatível com iterações de desenvolvimento rápidas. O objetivo deste artigo é identificar os desafios mais importantes na indústria e na academia referentes a ER ágil integrada a UCD.

\section{PALAVRA-CHAVE}

Usabilidade, Design Centrado no Usuário, Design Centrado no Humano, Interação Humano Computador, Desenvolvimento ágil de software, Engenharia de Requisito, Revisão Sistemática

\section{Introdução}

O envolvimento dos stakeholders e dos usuários é um fator crítico, para que um sistema tenha sucesso [3]. Consequentemente o aumento da popularização dos métodos ágeis, associado à pressão frequente por produtos que promovam uma melhor experiência de uso, está proporcionando uma troca entre as comunidades de interação humano-computador (IHC) e de desenvolvimento ágil [8]. A Engenharia de Requisitos (ER) é um processo fundamental para o sucesso dos projetos de software, pois fornece meios para descobrir, analisar e atender às necessidades dos usuários [2].

Realizar ER ágil centrada no usuário pode levar a desafios com os quais as organizações precisam lidar. No entanto, ainda há a necessidade de se fornecer uma visão geral dos desafios. $O$ objetivo principal deste trabalho é identificar os desafios mais importantes na indústria e na academia referentes a ER ágil integrada a UCD e assim construir uma compreensão compartilhada sobre esses desafios. A questão de pesquisa que foi colocada é "Quais os

Permission to reproduce or distribute, in whole or in part, material extracted from this work, verbatim, adapted or remixed, as well as the creation or production from the content of such work, is granted without fee for non-commercial use, provided that the original work is properly credited.

IHC 2019 - TRILHA PÔSTERES E DEMONSTRAÇÕES, Outubro 21-25, 2019, Vitória, Brasil. In Anais Estendidos do XVIII Simpósio Brasileiro sobre Fatores Humanos em Sistemas Computacionais. Porto Alegre: SBC.

(C) 2019 by the author(s), in accordance with the terms of the Creative Commons Attribution-NonCommercial 4.0 International Public License (CC BY-NC 4.0). desafios e limitações da engenharia de requisitos adotada em projetos ágeis integrados com UCD?".

\section{Referencial Teórico}

O Design Centrado no Usuário (User Centered Design (UCD), em inglês) é uma abordagem que visa envolver os usuários de maneira significativa e apropriada ao longo do desenvolvimento de um sistema, e a sua filosofia geral é colocar o usuário no centro do processo de design através do uso de métodos rigorosos [9]. Já os métodos ágeis são marcados por uma comunicação face-a-face, pela capacidade de se adaptar às mudanças e por um desenvolvimento de software incremental e iterativo [4]. A engenharia de requisitos fornece o mecanismo apropriado para entender aquilo que o cliente deseja [2]. Portanto, o envolvimento das stakeholders e do usuário final durante a ER ágil é essencial para estabelecer um ambiente colaborativo com ciclos de feedback constantes.

Para Caballero, Moreno e Seffah [1] os benefícios da UCD para o desenvolvimento ágil de software são inquestionáveis, logo a integração das abordagens Ágeis e Centradas no usuário não é apenas possível, como benéfica. Porém, é necessário aprofundar o conhecimento sobre a ER ágil e sua integração com UCD para fornecer aos profissionais mais detalhes sobre esse processo para que possam tomar decisões fundamentadas nessa direção.

\section{Metodologia}

Para conduzir este estudo, uma Revisão Sistemática da Literatura (RSL) foi realizada usando como referência os trabalhos de Kitchenham e Charters [6], de Dias e Silva [7] e de Falbo [5].

Para responder à questão da pesquisa deu-se início à execução da estratégia de pesquisa. Foram utilizadas fontes de busca automáticas na seleção dos estudos, sendo elas: ACM, IEEE Explore, Scopus, Science Direct e Web of Science. A partir do objetivo da pesquisa foram considerados três grupos de termos, para compor as palavras-chave, que são: Métodos Ágeis, Interação Humano-Computador e Engenharia de Requisitos. A seguir, é apresentado o termo de busca derivado: (( agile OR lean) AND ( "requirement engineering" OR ("human computer interaction" OR "human-centered" OR "user-centered" OR "user experience" OR usability))). Na seleção dos estudos, a fim de aumentar a relevância 
dos estudos selecionados, foram aplicados os critérios de inclusão e de exclusão.

A seleção dos estudos foi realizada em 6 (seis) etapas (figura 1). Inicialmente foram identificados 2111 artigos, sendo reduzidos para $14 \operatorname{artigos}^{1}$ (Figura 1).

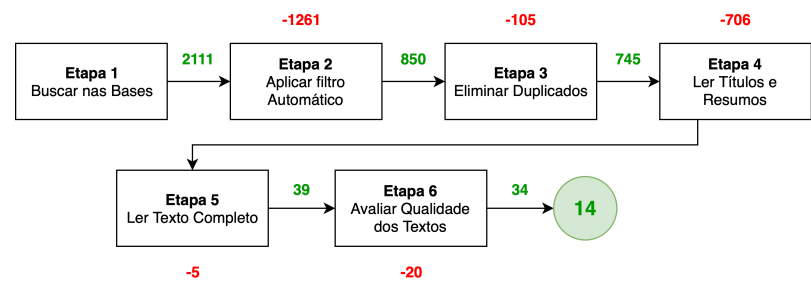

Figure 1: Processo de seleção de estudos para RSL

\section{Resultado e Discussão}

Os resultados da RSL fornecem informações importantes para pesquisadores e profissionais. Os resultados agora são discutidos em relação a pergunta da pesquisa.

Após a revisão e refinamentos sucessivos dos dados, foram identificadas categorias e subcategorias e seus respectivos desafios $^{2}$. Esta RSL apontou os desafios das atividades da engenharia de requisitos (ER) em projetos ágeis integrados com UCD, tendo como subcategorias de desafios: Levantamento de Requisito, Priorização de Requisito, Gestão de Requisitos e Documentação de Requisitos. Além dos desafios da ER, este trabalho obteve também resultados adicionais, pois foram identificados desafios referentes a outras categorias, como: A colaboração e cultura (onde as suas subcategorias de desafios identificadas foram: desafio referente à integração entre indivíduos da equipe, desafios para integração entre equipe ágeis e de UCD, desafio referente à sobrecarga de trabalho, Luta pelo Poder e desafios referentes ao Processo de Desenvolvimento); $\mathrm{O}$ Envolvimento do usuário (as subcategorias de desafios identificadas usuário foram: Expectativa do Usuário e Participação do Usuário); e Uso de Técnicas/Práticas (com as seguintes subcategorias de desafios identificadas: Aplicação das técnicas e Período de Tempo para a utilização de técnica/prátoca).

Através desta revisão sistemática, notou-se que as estratégias para levantar requisitos em projetos que abordam a integração do IHC e do Ágil mais utilizadas foram: User Story (8 ocorrências), Personas (7 ocorrências), Usability Test (5 ocorrências), Sprint Zero (5 ocorrências) e Prototyping/Prototype (11 ocorrências). Sendo também identificados alguns desafios ${ }^{3}$ presentes no uso dessas técnicas/práticas. Desta forma, o estudo indicou que a maioria dos desafios são decorrentes de problemas envolvendo a Colaboração e Cultura, a Engenheira de Requisito, mais especificamente na etapa de Levantamento de Requisitos, o Envolvimento do Usuário, e o Uso de Técnicas/Práticas. O estudo também indicou que algumas das técnicas/práticas, por exemplo,

\footnotetext{
${ }^{1}$ Link de acesso para os artigos selecionados: http://bit.ly/2kF9DIJ

${ }^{2}$ Link para a tabela de Categorias e Subcategorias de Desafios: http://bit.ly/2lIgstj
}

User Story e Prototyping/Prototype, usados para elicitar requisitos podem reforçar alguns desafios enfrentados na ER, como é o caso da negligência de RNF.

\section{Conclusão e Trabalhos Futuros}

Com os desafios levantados neste trabalho, pode-se perceber uma dificuldade inerente as organizações referente ao incentivo a colaboração entre as equipes de UCD e Ágeis. Por mais que o desenvolvimento híbrido torne o desenvolvimento mais centrado no usuário, ainda temos problemas com o envolvimento do usuário e do cliente, principalmente com a inclusão do usuário final no processo, e ainda existe a dificuldade em como utilizar o conhecimento adquirido através das técnicas na elicitação dos requisitos. Diante do exposto, pode-se concluir que por mais que os métodos ágeis possam ser combinados com UCD, e essa integração seja benéfica e facilite o envolvimento do usuário no processo, ainda não está claro como incorporar de forma eficiente o usuário e as práticas de UCD no ciclo de vida de desenvolvimento de software ágil, principalmente na ER ágil. Como tópicos de pesquisas futuras, é sugerido identificar formas de melhorar as práticas de comunicação e que permitam colaboração mais próxima entre profissionais de IHC, praticantes ágeis, usuários finais e cliente. Sugere-se ainda a realização de estudos de caso para melhor entender como ocorre a integração de IHC com ER ágil no ambiente real e verificar como a cultura da empresa pode influenciar o processo de desenvolvimento.

\section{AGRADECIMENTOS}

O presente trabalho foi realizado com apoio da Coordenação de Aperfeiçoamento de Pessoal de Nível Superior - Brasil (CAPES) Código de Financiamento 001.

\section{REFERÊNCIAS}

[1] L. Caballero, A. M. Moreno and A. Seffah. How Agile Developers Integrate User-Centered Design Into Their Processes: A Literature Review. International Journal Of Software Engineering And Knowledge Engineering, [s.l.], v. 26, n. 08, p.1175-120. 2016. DOI: http://dx.doi.org/10.1142/s0218194016500418.

[2] K. Elghariani; N. Kama. Review on Agile requirements engineering challenges. 2016 3rd International Conference On Computer And Information Sciences, p.507-512 .2016. DOI: http://dx.doi.org/10.1109/iccoins.2016.7783267.

[3] E. Schön; J. Thomaschewski; M. J. Escalona. Agile Requirements Engineering: A systematic literature review. Computer Standards \& Interfaces, [s.1.], v. 49, p.79-91, jan. 2017. DOI: http://dx.doi.org/10.1016/j.csi.2016.08.011.

[4] I. Sommerville (2011). Software Engineering, Boston, Massachusetts: Pearson Education.

[5] R. de A. Falbo. Mapeamento Sistemático. [s.1]: Universidade Federal do Espírito Santo, 2018. 25 p. https://inf.ufes.br/ falbo/files/MP/TP/Sobre_MS.pdf.

[6] B. Kitchenham and S. Charters, Guidelines for performing systematic literature reviews in software engineering. Tech. Rep. EBSE-2007-01, Keele University, 2007. DOI: http://citeseerx.ist.psu.edu/viewdoc/summary?doi=10.1.1.117.471.

[7] D. de S. Dias and M. F. da Silva. Como Escrever uma monografia: Manual de elaboração com exemplos e exercícios. São Paulo: Atlas, 2010. 152 p.

[8] C. Rosemberg and A. Schilling. Integrando IHC e métodos ágeis. In: Proceedings of the Companion Proceedings of the 10th Brazilian Symposium on Human Factors, 2011. p. 36-38. https://dl.acm.org/citation.cfm?id=2254534.

[9] S. Chamberlain; H. Sharp; N. Maiden. "Towards a Framework for Integrating Agile Development and User-Centred Design”. 2006 Extreme Programming And Agile Processes In Software Engineering. DOI: http://dx.doi.org/10.1007/11774129_15.

\footnotetext{
${ }^{3}$ Link para a tabela de desafios das Técnicas/Práticas: http://bit.ly/2kCIrdL
} 